Journal of Engineering and Applied Sciences 15 (2): 581-585, 2020

ISSN: 1816-949X

(C) Medwell Journals, 2020

\title{
Introduction to Cartesian, Tensor and Lexicographic Product of Bipolar Interval Valued Fuzzy Graph
}

\author{
${ }^{1}$ Wael Ahmad Al Zoubi, ${ }^{2}$ As’ad Mahmoud As’ad Alnaser, ${ }^{3}$ Hazem “Moh’d Said” Hatamleh, \\ ${ }^{4}$ Youssef Al Wadi and ${ }^{5}$ Mourad Oqla Massa'deh \\ 1, 2, 3, 5 Department of Applied Science, Ajloun University College, Al-Balqa Applied University, \\ Salt, Jordan \\ ${ }^{3}$ Department of Computer, Science College, Taibah University, Medina, Saudi Arabia \\ ${ }^{4}$ Department of Mathematics, Arab International University, Dara, Syria \\ ${ }^{5}$ Department of Mathematics, Faculty of Science, Taibah University, Medina, Saudi Arabia
}

\begin{abstract}
In this study, we discuss the product of bipolar, interval valued fuzzy graphs concept and we give some properties for them. We will study some corollaries on Cartesian product and define some properties on it. Tensor product, lexicographic product of bipolar interval valued fuzzy graph will also be defined.
\end{abstract}

Key words: Interval valued fuzzy set, bipolar interval valued fuzzy set, bipolar interval valued fuzzy graph, cartesian product, tensor product, lexicographic product

\section{INTRODUCTION}

A fuzzy set theory was introduced by Zadeh (1965). Fuzzy set theory has become a vigorous area of research in different disciplines including mathematics, physics, statistics, engineering and computer networks. In mathematics fuzzy groups, rings and graphs have been discussed by Massa'deh (2010a, b), Massa'deh and Ba'arah (2013) and Massa'deh and Gharaibeh (2011). Bipolar fuzzy set's concepts defined by Zhang (1998) is a generalization of fuzzy sets (Muthuraj et al., 2016; Muthuraj and Sridharan, 2012) discussed the concepts of bipolar fuzzy normal-subgraph (Massa'deh, 2017) introduced and studied bipolar fuzzy cosets. Ramya and Lavanya (2017) studied the edge contraction on bipolar fuzzy graphs (Massa'deh and Ba'arah, 2013) introduced the concept of degrees types in bipolar fuzzy graphs. Akram and Dudek (2011) extended the fuzzy set theory to interval-valued fuzzy sets, in addition (Ramprasad et al., 2016) discussed interval valued fuzzy graphs, also (Massa’deh, 2017) discussed regular, degree of vertex, strong, complete interval valued fuzzy graphs (Rashmanlou and Pal, 2013a-c; Rashmanlou and Jun, 2013). Mishra et al. studied bipolar interval valued fuzzy graphs (Mishra and Pal, 2016). In this study, we gave and studied the product of two bipolar interval-valued fuzzy graph concepts and discussed some of their properties, we defined also tensor product and lexicographic of bipolar interval-valued fuzzy graphs.

\section{MATERIALS AND METHODS}

\section{Preliminaries}

Definition 2.1 (Zadeh 1965): A fuzzy set $\mu$ is a mapping from $\mathrm{X}$ to $[0,1]$.

Definition 2.2 (Massa'deh and Gharaibeh (2011): A fuzzy graph $G$ is a pair of functions $G=(\lambda, \mu)$ where, $\lambda$ is a fuzzy subset of a non-empty set $X$ and $\mu$ is a symmetric fuzzy relation on $\lambda$ this means that $\mu(\mathrm{xy}) \leq \max \{\lambda(\mathrm{x}), \lambda$ (y) $\}$. The underlying crisp graph of $G=(\lambda, \mu)$ is denoted by $\mathrm{G}^{*}=(\mathrm{V}, \mathrm{E})$ where $\mathrm{E} \subseteq \mathrm{V} \mathrm{X} \mathrm{V}$.

Definition 2.3 (Massa'deh and Ba'arah (2013): Let $G=(\lambda, \mu)$ be a fuzzy graph, the degree of a vertex $a \in G$ is defined by:

$$
\mathrm{D}_{\mathrm{G}}(\mathrm{a})=\sum \mathrm{a} \neq \mathrm{b} \mu(\mathrm{ab})=\sum \mathrm{ab} \in \mathrm{E} \mu(\mathrm{ab})
$$

Definition 2.4 (Massa'deh and Ba'arah (2013): The order of a fuzzy graph $G$ is defined by $O(G)=\Sigma_{a b \in E} \lambda(a)$ :

Definition 2.5 Zhang (1998): Let X be a non-empty set. A bipolar fuzzy set $\mu$ in $X$ is an object having the form $\mu$ $=\left\{\left(\mathrm{x}, \mu^{+}(\mathrm{x}), \mu^{-}(\mathrm{x})\right) ; \mathrm{x} \in \mathrm{X}\right\}$ where $\mu^{+}(\mathrm{x}): \mathrm{X} \rightarrow[0,1]$ and $\mu^{-}$ (x): $\mathrm{X} \rightarrow[-1,0]$ are mapping. Here, $\mu^{+}(\mathrm{x})$ is the positive membership value which denotes the satisfaction degree of an element $\mathrm{x} \in \mu$ and $\mu^{-}(\mathrm{x})$ is the negative membership value which denotes the satisfaction degree to some implicit counter property of an element $\mathrm{x} \in \mu$. If for any 
$\mathrm{x} \in \mu, \mu^{+}(\mathrm{x}) \neq 0$ and $\mu^{-}(\mathrm{x})=0$, it is the situation that $\mathrm{x}$ has only positive satisfaction for $\mu$, if for any $x \in \mu, \mu^{+}(x)=0$ and $\mu^{-}(x) \neq 0$ then the situation that $x$ does not satisfy the property of $\mu$ but somewhat satisfies the counter property of $\mu$. It is possible for an element $\mathrm{x}$ for which $\mu^{+}$ (x) $=0$ and $\mu^{-}(\mathrm{x})=0$ then we say that the satisfaction property of an element overlaps with its counter satisfaction property over some portion, we shall use the symbol $\mu\left(\mu^{+}, \mu^{-}\right)$for the bipolar fuzzy set $\mu=\left\{\left(x, \mu^{+}(x)\right.\right.$, $\left.\left.\mu^{-}(\mathrm{x})\right) ; \mathrm{x} \in \mu\right\}$.

Definition 2.6 (Zhang, 1998): For every two bipolar fuzzy sets $\mu=\left(\mu^{+}, \mu^{-}\right)$and $\lambda=\left(\lambda^{+}, \lambda^{-}\right)$in A, we define:

$$
\begin{aligned}
& (\mu \cap \lambda)(a)=\left(\min \left(\mu^{+}(a), \lambda^{+}(a)\right), \max \left(\mu^{-}(a), \lambda^{-}(a)\right)\right. \\
& (\mu \cup \lambda)(a)=\left(\max \left(\mu^{+}(a), \lambda^{+}(a)\right), \min \left(\mu^{-}(a), \lambda^{-}(a)\right)\right.
\end{aligned}
$$

For all $\mathrm{a} \in \mathrm{A}$ :

Definition 2.7 (Zhang, 1998): Let $\mu=\left(\mu^{+}, \mu^{-}\right)$and $\lambda=$ $\left(\lambda^{+}, \lambda^{-}\right)$be two bipolar fuzzy sets on $A$. If $\delta=\mu \times \lambda$ is any relation on A then $\delta=\left(\delta^{+}, \delta^{-}\right)$is called a bipolar fuzzy relation from $\mu=\left(\mu^{+}, \mu^{-}\right)$on $\lambda=\left(\lambda^{+}, \lambda^{-}\right)$where $\delta^{+}$(a, b) $\leq \min \left\{\mu^{+}(\mathrm{a}), \lambda^{+}(\mathrm{b})\right\}$ and $\delta^{-}(\mathrm{a}, \mathrm{b}) \geq \max \left\{\mu^{-}(\mathrm{a}), \lambda^{-}\right.$(b) $\}$ for all $a \in \mu$ and $b \in \lambda$.

Throughout this study, $G$ is a crisp graph, $F_{G}$ is a fuzzy graph, $\mathrm{BF}_{\mathrm{G}}$ is a bipolar fuzzy graph, $\mathrm{IVF}_{\mathrm{G}}$ is an interval-valued fuzzy graph and $\mathrm{BIVF}_{\mathrm{G}}$ is a bipolar interval-valued fuzzy graph.

Let $A=\left\{a_{1}, a_{2}, \ldots, a_{n}\right\}$ be any set and $[0,1]$ be the set of all closed sub-intervals of the interval $[0,1],[-1,0]$ be the set of all closed sub-intervals of the interval $[-1,0]$ and elements of these sets are denoted by uppercase letters. If $\mu \in \mathrm{C}[0,1]$ or $\mathrm{K}[-1,0]$ then it can be represented as $\mu=\left[\mu_{\mathrm{L}}, \mu_{\mathrm{u}}\right]$ where $\mu_{\mathrm{L}}$ and $\mu_{\mathrm{u}}$ are the lower and upper limit of $\mu$.

Definition 2.8 (Mishra and Pal, 2016) BIVF subset is given by $\mu=\left\{<a, \mu^{+}(a), \mu^{-}(a)>, a \in A\right\}$ where $\mu^{+}: A \rightarrow C[0,1], \mu^{-}: A \rightarrow K[-1,0]$. The intervals $\mu^{+}(a)$ and $\mu^{-}$(a) denote the degree of membership and the degree of non-membership of the element a to the set, where $\mu^{+}(a)=\left[\mu_{L}^{+}(a), \mu_{U}^{+}(a)\right]$ and $\mu^{-}(a)=\left[\mu_{L}^{-}\right.$ (a), $\mu^{-}$(a)].

Definition 2.9 (Mishra and Pal, 2016): By bipolar interval-valued fuzzy set on $\mathrm{V}$ and $\lambda=\left[\lambda^{-}, \lambda^{+}\right]$is a bipolar interval-valued fuzzy relation on $\mathrm{E}$ such that:

- $\quad \lambda^{-}(\mathrm{ab}) \leq \min \left\{\mu^{-}(\mathrm{a}), \mu^{-}(\mathrm{b})\right\}$

- $\quad \lambda^{+}(\mathrm{ab}) \leq \min \left\{\mu^{+}(\mathrm{a}), \mu^{+}(\mathrm{b})\right\}$ for all $\mathrm{ab} \in \mathrm{E}$
Definition 2.10 (Ramya and Lavanya, 2017): By a bipolar fuzzy graph $\mathrm{BF}_{\mathrm{G}}$ of $\mathrm{G}=(\mathrm{V}, \mathrm{E})$ we main a pair $(\delta$, $\gamma$ ) where $\delta=\left(\delta^{+}, \delta^{-}\right)$is a bipolar fuzzy set on $\mathrm{V}$ and $\gamma=$ $\left(\gamma^{+}, \gamma^{-}\right)$is a bipolar fuzzy relation on $\mathrm{E} \subseteq \mathrm{V} X \mathrm{~V}$ such that $\gamma^{+}(\mathrm{ab}) \leq \min \left\{\delta^{+}(\mathrm{a}), \delta^{+}(\mathrm{b})\right\}$ and $\gamma^{-}(\mathrm{ab}) \leq \max \left\{\delta^{-}(\mathrm{a}), \delta^{-}\right.$ (b) $\}$ for all $a, b \in V$ and $a b \in E$.

Definition 2.11 (Rashmanlou and Pal 2013): Let $\mathrm{G}_{1}=\left(\mathrm{V}_{1}, \mathrm{E}_{1}\right)$ and $\mathrm{G}_{2}=\left(\mathrm{V}_{2}, \mathrm{E}_{2}\right)$ be two simple graphs, we can construct several new graphs. The first construction called the Cartesian product of $G_{1}$ and $G_{2}$ gives a graph $\mathrm{G}_{1} \cdot \mathrm{G}_{2}=(\mathrm{V}, \mathrm{E})$ with $\mathrm{V}=\mathrm{V}_{1} \mathrm{X} \mathrm{V_{2 }}$ and $\mathrm{E}=$ $\left\{(\mathrm{a}, \mathrm{b})(\mathrm{a}, \mathrm{c}) ; \mathrm{a} \in \mathrm{V}_{1}, \mathrm{bc} \in \mathrm{E}_{2}\right\} U\left\{\left(\mathrm{~d}_{1}, \mathrm{e}\right)\left(\mathrm{d}_{2}, \mathrm{l}\right) ; \mathrm{d}_{1} \mathrm{~d}_{2} \in \mathrm{E}_{1}\right.$, $\left.\mathrm{e} \in \mathrm{V}_{2}\right\}$.

Throughout this study, we assume $\mathrm{C}[0,1]$ be the set of all closed sub-intervals of the interval $[0,1]$ and $\mathrm{K}[-1,0]$ is the set of all closed sub-intervals of the interval $[-1,0]$.

\section{RESULTS AND DISCUSSION}

Definition 3.1: A Bipolar Interval-valued Fuzzy Graph $\mathrm{BIVF}_{\mathrm{G}}$ with underlying graph $\mathrm{G}=(\mathrm{V}, \mathrm{E})$ is defined to be a pair $(\lambda, \mu)$ where:

The function $\lambda^{+}: \mathrm{V} \rightarrow \mathrm{C}[0,1]$ and $\lambda^{-}: \mathrm{V} \rightarrow \mathrm{K}[-1,0]$ denote satisfaction degree interval and the satisfaction degree interval to some implicit counter-property of an element $\mathrm{a} \in \lambda$, respectively.

The function $\mu^{+}: \mathrm{E} \subset \mathrm{V} X \mathrm{~V} \rightarrow \mathrm{C}[0,1]$ and $\mu^{+}: \mathrm{E} \subset \mathrm{V} X$ $\mathrm{V} \rightarrow \mathrm{K}[-1,0]$ are defined by $\mu_{\mathrm{L}}^{+}(\mathrm{a}, \mathrm{b}) \leq \min \left\{\lambda_{\mathrm{L}}^{+}(\mathrm{a}), \lambda_{\mathrm{L}}^{+}\right.$ (b) $\}$ and $\mu^{-}{ }_{L}(a, b) \geq \max \left\{\lambda^{-}{ }_{L}(a), \lambda_{L}^{-}\right.$(b) $\} \mu_{U}^{+}$(a, b) $\leq \min$ $\left\{\mu_{U}^{+}(a), \mu_{U}^{+}(b)\right\}$ and $\mu^{-}(a, b) \geq \max \left\{\mu^{-}(a), \mu_{U}^{-}(b)\right\}$ for all $a b \in \mathrm{E}$.

Example 3.2: Consider a Bipolar Interval-valued Fuzzy graph $\mathrm{BIF}_{\mathrm{G}}$ where, $\lambda=(\mathrm{a},[0.2,0.6],[-0.7,-0.5]),(\mathrm{b},[0.3$, $0.5],[-0.9,-0.3]),(c,[0.5,0.4],[-0.6,-0.5]),(d,[0.1,0.7]$, $[-0.8,-0.2])$. Then the corresponding $\mathrm{BIVF}_{\mathrm{G}}$ is shown in Fig. 1.

Definition 3.3: Let $G_{1}=\left(\lambda_{1}, \mu_{1}\right)$ and $G_{2}=\left(\lambda_{2}, \mu_{2}\right)$ be two bipolar interval valued fuzzy graph of the graph is $G_{1}^{*}=\left(V_{1}, E_{1}\right)$ and $G_{2}^{*}=\left(V_{2}, E_{2}\right)$ then the Cartesian product $\mathrm{G}=\left(\mathrm{G}_{1} \times \mathrm{G}_{2}\right)$ is defined as pain $\left(\lambda_{1}, \lambda_{2}, \mu_{1} \times \mu_{2}\right)$ such that:

$$
\begin{aligned}
& \left(\lambda_{1 \mathrm{~L}}^{+} \times \lambda_{2 \mathrm{~L}}^{+}\right)(\mathrm{a}, \mathrm{b})=\min \left\{\lambda_{1 \mathrm{~L}}^{+}(\mathrm{a}), \lambda_{2 \mathrm{~L}}^{+}(\mathrm{b})\right\} \\
& \left(\lambda_{1 \mathrm{U}}^{+} \times \lambda_{2 \mathrm{U}}^{+}\right)(\mathrm{a}, \mathrm{b})=\min \left\{\lambda_{1 \mathrm{U}}^{+}(\mathrm{a}), \lambda_{2 \mathrm{U}}^{+}(\mathrm{b})\right\} \\
& \left(\lambda_{1 \mathrm{~L}}^{-} \times \lambda_{2 \mathrm{~L}}^{-}\right)(\mathrm{a}, \mathrm{b})=\max \left\{\lambda_{1 \mathrm{~L}}^{-}(\mathrm{a}), \lambda_{2 \mathrm{~L}}^{-}(\mathrm{b})\right\} \\
& \left(\lambda_{1 \mathrm{U}}^{-} \times \lambda_{2 \mathrm{U}}^{-}\right)(\mathrm{a}, \mathrm{b})=\max \left\{\lambda_{1 \mathrm{U}}^{-}(\mathrm{a}), \lambda_{2 \mathrm{U}}^{-}(\mathrm{b})\right\} \\
& \text { For all }(\mathrm{a}, \mathrm{b}) \in \mathrm{V}
\end{aligned}
$$




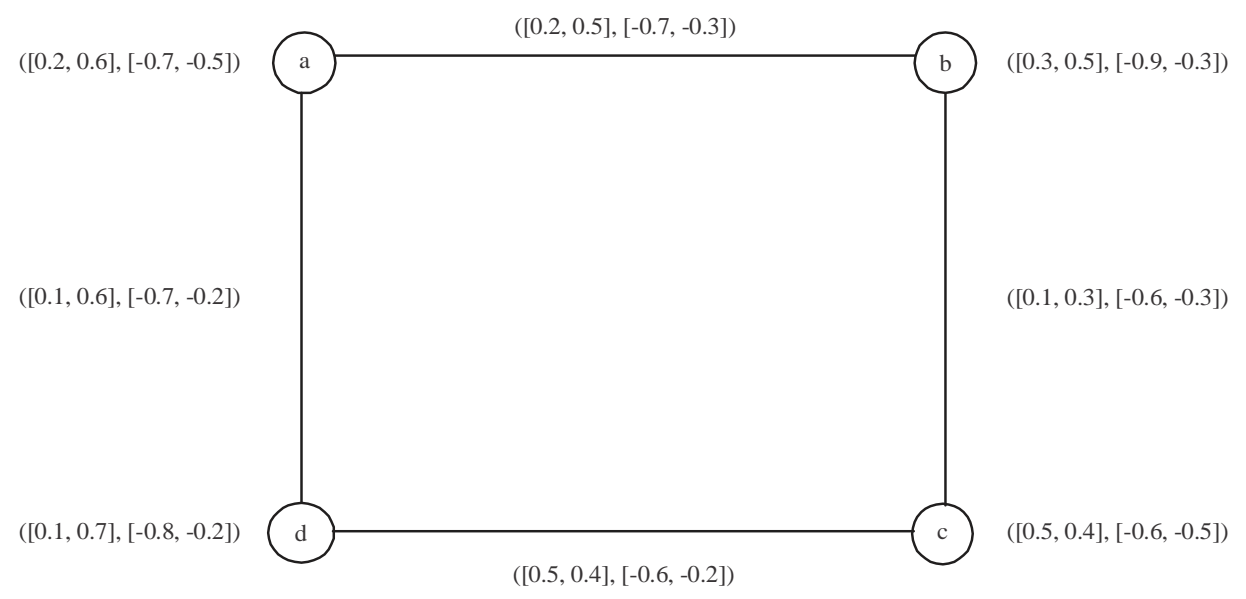

Fig. 1: Bipolar interval-valued fuzzy graph

$$
\begin{aligned}
& \left(\mu_{1 \mathrm{~L}}^{+} \times \mu_{2 \mathrm{~L}}^{+}\right)((\mathrm{a}, \mathrm{b})(\mathrm{a}, \mathrm{c}))=\min \left\{\lambda_{1 \mathrm{~L}}^{+}(\mathrm{a}), \mu_{2 \mathrm{~L}}^{+}(\mathrm{bc})\right\} \\
& \left(\mu_{1 \mathrm{U}}^{+} \times \mu_{2 \mathrm{U}}^{+}\right)((\mathrm{a}, \mathrm{b})(\mathrm{a}, \mathrm{c}))=\min \left\{\lambda_{\mathrm{LL}}^{+}(\mathrm{a}), \mu_{2 \mathrm{U}}^{+}(\mathrm{bc})\right\} \\
& \left(\mu_{1 \mathrm{~L}}^{-} \times \mu_{2 \mathrm{~L}}^{-}\right)((\mathrm{a}, \mathrm{b})(\mathrm{a}, \mathrm{c}))=\max \left\{\lambda_{\mathrm{IL}}^{-}(\mathrm{a}), \mu_{2 \mathrm{~L}}^{-}(\mathrm{bc})\right\} \\
& \left(\mu_{1 \mathrm{U}}^{-} \times \mu_{2 \mathrm{U}}^{-}\right)((\mathrm{a}, \mathrm{b})(\mathrm{a}, \mathrm{c}))=\max \left\{\lambda_{1 \mathrm{U}}^{-}(\mathrm{a}), \mu_{2 \mathrm{U}}^{-}(\mathrm{bc})\right\} \\
& \text { For all a } \in \mathrm{V} \text { and bc } \in \mathrm{E}_{2} \\
& \left(\mu_{1 \mathrm{~L}}^{+} \times \mu_{2 \mathrm{~L}}^{+}\right)((\mathrm{a}, \mathrm{b})(\mathrm{c}, \mathrm{b}))=\min \left\{\mu_{1 \mathrm{~L}}^{+}(\mathrm{ac}), \lambda_{2 \mathrm{~L}}^{+}(\mathrm{b})\right\} \\
& \left(\mu_{1 \mathrm{U}}^{+} \times \mu_{2 \mathrm{U}}^{+}\right)((\mathrm{a}, \mathrm{b})(\mathrm{c}, \mathrm{b}))=\min \left\{\mu_{1 \mathrm{U}}^{+}(\mathrm{ac}), \lambda_{2 \mathrm{~L}}^{+}(\mathrm{b})\right\} \\
& \left(\mu_{1 \mathrm{~L}}^{-} \times \mu_{2 \mathrm{~L}}^{-}\right)((\mathrm{a}, \mathrm{b})(\mathrm{c}, \mathrm{b}))=\max \left\{\mu_{1 \mathrm{~L}}^{-}(\mathrm{ac}), \lambda_{2 \mathrm{~L}}^{-}(\mathrm{b})\right\} \\
& \left(\mu_{1 \mathrm{U}}^{-} \times \mu_{2 \mathrm{U}}^{-}\right)((\mathrm{a}, \mathrm{b})(\mathrm{c}, \mathrm{b}))=\max \left\{\mu_{1 \mathrm{U}}^{-}(\mathrm{a}), \lambda_{2 \mathrm{U}}^{-}(\mathrm{b})\right\} \\
& \text { For all b } \in \mathrm{V} \text { and ac } \in \mathrm{E}_{1}
\end{aligned}
$$

Definition 3.4: If $G_{1}=\left(\lambda_{1}, \mu_{1}\right)$ and $G_{2}=\left(\lambda_{2}, \mu_{2}\right)$ are two bipolar interval valued fuzzy graph of $\mathrm{G}_{1}^{*}=\left(\mathrm{V}_{1}, \mathrm{E}_{1}\right)$ and $\mathrm{G}_{2}{ }_{2}=\left(\mathrm{V}_{2}, \mathrm{E}_{2}\right)$, respectively then the lexicographic product $\mathrm{G}_{1}{ }^{*} \mathrm{G}_{2}$ is defined as a pair $(\lambda, \mu)$ where $\lambda=\left(\lambda^{+}, \lambda^{-}\right)$and $\mu$ $=\left(\mu^{+}, \mu^{-}\right)$are bipolar interval valued fuzzy sets on $\mathrm{V}=$ $\mathrm{V}_{1} \times \mathrm{V}_{2}$ and $\mathrm{E}=\left\{(\mathrm{a}, \mathrm{b})(\mathrm{a}, \mathrm{c}) ; \mathrm{a} \in \mathrm{V}_{1},(\mathrm{~b}, \mathrm{c}) \in \mathrm{E}_{2}\right\} \cup\{(\mathrm{x}, \mathrm{y})$ $\left.(\mathrm{z}, \mathrm{w}) ; \mathrm{xz} \in \mathrm{E}_{1}, \mathrm{yw} \in \mathrm{E}_{2}\right\}$, respectively which satisfies the following conditions:

$$
\begin{aligned}
& \left(\lambda_{1 \mathrm{~L}}^{+} * \lambda_{2 \mathrm{~L}}^{+}\right)(\mathrm{x}, \mathrm{y})=\min \left\{\lambda_{\mathrm{IL}}^{+}(\mathrm{x}), \lambda_{2 \mathrm{~L}}^{+}(\mathrm{y})\right\} \\
& \left(\lambda_{1 \mathrm{U}}^{+} * \lambda_{2 \mathrm{U}}^{+}\right)(\mathrm{x}, \mathrm{y})=\min \left\{\lambda_{1 \mathrm{U}}^{+}(\mathrm{x}), \lambda_{2 \mathrm{U}}^{+}(\mathrm{y})\right\} \\
& \left(\lambda_{\mathrm{IL}}^{-} * \lambda_{2 \mathrm{~L}}^{-}\right)(\mathrm{x}, \mathrm{y})=\max \left\{\lambda_{\mathrm{IL}}^{-}(\mathrm{x}), \lambda_{2 \mathrm{~L}}^{-}(\mathrm{y})\right\} \\
& \left(\lambda_{1 \mathrm{U}}^{-} * \lambda_{2 \mathrm{U}}^{-}\right)(\mathrm{x}, \mathrm{y})=\max \left\{\lambda_{1 \mathrm{U}}^{-}(\mathrm{x}), \lambda_{2 \mathrm{U}}^{-}(\mathrm{y})\right\} \\
& \text { For all }(\mathrm{x}, \mathrm{y}) \in \mathrm{V}_{1} \times \mathrm{V}_{2} \\
& \left(\mu_{1 \mathrm{~L}}^{+} *^{+} \mu_{2 \mathrm{~L}}^{+}\right)((\mathrm{a}, \mathrm{b})(\mathrm{a}, \mathrm{c}))=\min \left\{\lambda_{1 \mathrm{~L}}^{+}(\mathrm{a}), \mu_{2 \mathrm{~L}}^{+}(\mathrm{bc})\right\} \\
& \left(\mu_{1 \mathrm{U}}^{+}{ }^{*} \mu_{2 \mathrm{U}}^{+}\right)((\mathrm{a}, \mathrm{b})(\mathrm{a}, \mathrm{c}))=\min \left\{\lambda_{1 \mathrm{U}}^{+}(\mathrm{a}), \mu_{2 \mathrm{U}}^{+}(\mathrm{ac})\right\} \\
& \left(\mu_{1 \mathrm{~L}}^{-}{ }^{*} \mu_{2 \mathrm{~L}}^{-}\right)((\mathrm{a}, \mathrm{b})(\mathrm{a}, \mathrm{c}))=\max \left\{\lambda_{1 \mathrm{~L}}^{-}(\mathrm{a}), \mu_{2 \mathrm{~L}}^{-}(\mathrm{ac})\right\} \\
& \left(\mu_{1 \mathrm{U}}^{-}{ }^{*} \mu_{2 \mathrm{U}}^{-}\right)((\mathrm{a}, \mathrm{b})(\mathrm{a}, \mathrm{c}))=\max \left\{\lambda_{1 \mathrm{~L}}^{-}(\mathrm{a}), \mu_{2 \mathrm{U}}^{-}(\mathrm{ac})\right\} \\
& \text { For all } \mathrm{a} \in \mathrm{V}_{1} \text { and } \mathrm{bc} \in \mathrm{E}_{2}
\end{aligned}
$$

$$
\begin{aligned}
& \left(\mu_{1 \mathrm{~L}}^{+}{ }^{*} \mu_{2 \mathrm{~L}}^{+}\right)((\mathrm{x}, \mathrm{y})(\mathrm{z}, \mathrm{w}))=\min \left\{\mu_{1 \mathrm{~L}}^{+}(\mathrm{xz}), \mu_{2 \mathrm{~L}}^{+}(\mathrm{yw})\right\} \\
& \left(\mu_{1 \mathrm{U}}^{+}{ }^{*} \mu_{2 \mathrm{U}}^{+}\right)((\mathrm{x}, \mathrm{y})(\mathrm{z}, \mathrm{w}))=\min \left\{\mu_{1 \mathrm{U}}^{+}(\mathrm{xz}), \mu_{2 \mathrm{U}}^{+}(\mathrm{yw})\right\} \\
& \left(\mu_{1 \mathrm{~L}}^{-} \mu^{-}\right)((\mathrm{x}, \mathrm{y})(\mathrm{z}, \mathrm{w}))=\max \left\{\mu_{1 \mathrm{~L}}^{-}(\mathrm{xz}), \mu_{2 \mathrm{~L}}^{-}(\mathrm{yw})\right\} \\
& \left(\mu_{1 \mathrm{U}}^{-}{ }^{*} \mu_{2 \mathrm{U}}^{-}\right)((\mathrm{x}, \mathrm{y})(\mathrm{z}, \mathrm{w}))=\max \left\{\mu_{1 \mathrm{U}}^{-}(\mathrm{xz}), \mu_{2 \mathrm{U}}^{-}(\mathrm{yw})\right\} \\
& \text { For all } \mathrm{xz} \in \mathrm{E}_{1} \text { and } \mathrm{yw} \in \mathrm{E}_{2}
\end{aligned}
$$

Definition 3.5: Let $G_{1}=\left(\lambda_{1}, \mu_{1}\right)$ and $G_{2}=\left(\lambda_{2}, \mu_{2}\right)$ be two bipolar interval valued fuzzy graph of $G_{1}^{*}=\left(V_{1}\right.$, $\left.E_{1}\right)$ and $G_{2}^{*}=\left(V_{2}, E_{2}\right)$, respectively then the tensor product $G_{1} \otimes G_{2}$ is defined as a pain $(\lambda, \mu)$ where $\lambda$ and $\mu$ are bipolar interval valued fuzzy sets on 12 $\mathrm{V}=\mathrm{V}^{\prime} \mathrm{V}$ and $\mathrm{V}=\mathrm{V}_{1} \times \mathrm{V}_{2}$ and $\mathrm{E}=\{(\mathrm{a}, \mathrm{b})(\mathrm{c}, \mathrm{d})$; (a, c) $\in \mathrm{E}_{1}$, (b, d) $\left.\in \mathrm{E}_{2}\right\}$, respectively which satisfies the following axioms:

$$
\begin{aligned}
& \left(\lambda_{1 \mathrm{~L}}^{+} \otimes \lambda_{2 \mathrm{~L}}^{+}\right)(\mathrm{a}, \mathrm{b})=\min \left\{\lambda_{1 \mathrm{~L}}^{+}(\mathrm{a}), \lambda_{2 \mathrm{~L}}^{+}(\mathrm{b})\right\} \\
& \left(\lambda_{1 \mathrm{U}}^{+} \otimes \lambda_{2 \mathrm{U}}^{+}\right)(\mathrm{a}, \mathrm{b})=\min \left\{\lambda_{1 \mathrm{U}}^{+}(\mathrm{a}), \lambda_{2 \mathrm{U}}^{+}(\mathrm{b})\right\} \\
& \left(\lambda_{1 \mathrm{~L}}^{-} \otimes \lambda_{2 \mathrm{~L}}^{-}\right)(\mathrm{a}, \mathrm{b})=\max \left\{\lambda_{1 \mathrm{~L}}^{-}(\mathrm{a}), \lambda_{2 \mathrm{~L}}^{-}(\mathrm{b})\right\} \\
& \left(\lambda_{1 \mathrm{U}}^{-} \otimes \lambda_{2 \mathrm{U}}^{-}\right)(\mathrm{a}, \mathrm{b})=\max \left\{\lambda_{1 \mathrm{U}}^{-}(\mathrm{a}), \lambda_{2 \mathrm{U}}^{-}(\mathrm{b})\right\} \\
& \text { For all }(\mathrm{a}, \mathrm{b}) \in \mathrm{V}_{1} \times \mathrm{V}_{2}
\end{aligned}
$$

$$
\begin{aligned}
& \left(\mu_{1 \mathrm{~L}}^{+} \otimes \mu_{2 \mathrm{~L}}^{+}\right)((\mathrm{a}, \mathrm{b})(\mathrm{c}, \mathrm{d}))=\min \left\{\mu_{1 \mathrm{~L}}^{+}(\mathrm{ac}), \mu_{2 \mathrm{~L}}^{+}(\mathrm{bd})\right\} \\
& \left(\mu_{1 \mathrm{U}}^{+} \otimes \mu_{2 \mathrm{U}}^{+}\right)((\mathrm{a}, \mathrm{b})(\mathrm{c}, \mathrm{d}))=\min \left\{\mu_{1 \mathrm{U}}^{+}(\mathrm{ac}), \mu_{2 \mathrm{U}}^{+}(\mathrm{bd})\right\} \\
& \left(\mu_{1 \mathrm{~L}}^{-} \otimes \mu_{2 \mathrm{~L}}^{-}\right)((\mathrm{a}, \mathrm{b})(\mathrm{c}, \mathrm{d}))=\max \left\{\mu_{1 \mathrm{~L}}^{-}(\mathrm{ac}), \mu_{2 \mathrm{~L}}^{-}(\mathrm{bd})\right\} \\
& \left(\mu_{1 \mathrm{U}}^{-} \otimes \mu_{2 \mathrm{U}}^{-}\right)((\mathrm{a}, \mathrm{b})(\mathrm{c}, \mathrm{d}))=\max \left\{\mu_{1 \mathrm{U}}^{-}(\mathrm{ac}), \mu_{2 \mathrm{U}}^{-}(\mathrm{bd})\right\} \\
& \text { For all ac } \in \mathrm{E}_{1} \text { and bd } \in \mathrm{E}_{2}
\end{aligned}
$$

Definition 3.6: If $G_{1}, G_{2}$ are two bipolar interval valued fuzzy graph and $G=G_{1} \times G_{2}$ is the Cartesian product of $G_{1}$ and $G_{2}$ then for any vertex $(a, b) \in V_{1} \times V_{2}$, we define the degree of $(a, b)$ as: 


$$
\begin{aligned}
& \mathrm{D}_{\mathrm{G}}^{+}(\mathrm{a}, \mathrm{b})=\sum_{(\mathrm{a}, \mathrm{b})(\mathrm{c}, \mathrm{d}) \mathrm{E}}\left(\mu_{\mathrm{u}}^{+} \times \mu_{\mathrm{uv}}^{+}\right)((\mathrm{a}, \mathrm{b})(\mathrm{c}, \mathrm{d}))- \\
& \sum_{(\mathrm{a}, \mathrm{b})(\mathrm{c}, \mathrm{d}) \in \mathrm{E}}\left(\mu_{\mathrm{iv}}^{-} \times \mu_{2 \mathrm{i}}^{-}\right)((\mathrm{a}, \mathrm{b})(\mathrm{c}, \mathrm{d}))= \\
& \sum_{a=c, b d \in E_{2}} \min \left\{\lambda_{1 \mathrm{U}}^{+}(\mathrm{a}), \mu_{2 \mathrm{U}}^{+}(\mathrm{bd})\right\}^{+} \\
& \sum_{b=d, a c \in E_{1}} \min \left\{\lambda_{2 \mathrm{U}}^{+}(\mathrm{b}), \mu_{1 \mathrm{U}}^{+}(\mathrm{ac})\right\}- \\
& \left\{\sum_{\mathrm{a}=\mathrm{c}, \mathrm{bd \in E_{2 }}} \max \left\{\begin{array}{l}
\lambda_{\mathrm{iU}}^{-}(\mathrm{a}), \\
\mu_{\mathrm{zU}}^{-}(\mathrm{bd})
\end{array}\right\}+\sum_{\mathrm{b}=\mathrm{d}, \mathrm{accE} \mathrm{E}_{1}} \max \left\{\begin{array}{l}
\lambda_{2 \mathrm{v}}^{-}(\mathrm{b}), \\
\mu_{\mathrm{IU}}^{-}(\mathrm{ac})
\end{array}\right\}\right\}
\end{aligned}
$$

Similarly:

$$
\begin{aligned}
& D_{\mathrm{G}}^{-}(\mathrm{a}, \mathrm{b})=\sum_{(\mathrm{a}, \mathrm{b})(\mathrm{c}, \mathrm{d}) \in \mathrm{E}}\left(\mu_{\Perp \mathrm{L}}^{+} \times \mu_{2 \mathrm{~L}}^{+}\right)((\mathrm{a}, \mathrm{b})(\mathrm{c}, \mathrm{d}))- \\
& \sum_{(\mathrm{a}, \mathrm{b})(\mathrm{c}, \mathrm{d}) \in \mathrm{E}}\left(\mu_{\mathrm{it}}^{-} \times \mu_{2 \mathrm{c}}^{-}\right)((\mathrm{a}, \mathrm{b})(\mathrm{c}, \mathrm{d}))= \\
& \sum_{a=c, b d E_{2}} \min \left\{\begin{array}{l}
\lambda_{1 L}^{+}(a), \\
\mu_{2 L}^{+}(b d)
\end{array}\right\}+\sum_{b=d, a c \in E_{1}} \min \left\{\begin{array}{l}
\lambda_{2 L}^{+}(b), \\
\mu_{1 L}^{+}(a b)
\end{array}\right\}- \\
& \left\{\sum_{a=c, b d E_{2}} \max \left\{\begin{array}{l}
\lambda_{1 \mathrm{~L}}^{-}(\mathrm{a}), \\
\mu_{\mathrm{LL}}^{-}(\mathrm{bd})
\end{array}\right\}+\sum_{\mathrm{b}=\mathrm{d}, \mathrm{ac \in E}} \max \left\{\begin{array}{l}
\lambda_{2 \mathrm{~L}}^{-}(\mathrm{b}), \\
\mu_{\mathrm{IL}}^{-}(\mathrm{ac})
\end{array}\right\}\right\}
\end{aligned}
$$

Example 3.7: Consider a bipolar interval valued fuzzy graphs $G_{1}, G_{2}$ and $G_{1} \times G_{2}$ in Fig. 2.

Definition 3.7: A Cartesian product of graph is:

- Strong, if $\mathrm{D}^{-}{ }_{\mathrm{G}} \leq 0$ and $\mathrm{D}^{+}{ }_{\mathrm{G}}>0$

- Week, if $\mathrm{D}^{-}{ }_{\mathrm{G}}<0$ and $\mathrm{D}_{\mathrm{G}}^{+} \geq 0$

- Super strong, if $\mathrm{D}^{-}{ }_{\mathrm{G}}<0$ and $\mathrm{D}_{\mathrm{G}}>0$

- Very week, if $\mathrm{D}_{\mathrm{G}_{\mathrm{G}}}^{-}<0$ and $\mathrm{D}_{\mathrm{G}}^{+}>0$

Theorem 3.8: Let $G$ be the Cartesian product of two bipolar interval valued fuzzy graphs then $G$ is super strong if:

- $\quad \operatorname{Min}\left\{\lambda^{+}{ }_{\mathrm{iL}}\left(\mathrm{a}_{\mathrm{i}}\right)\right\} \geq \max \left\{\lambda^{-}{ }_{\mathrm{iL}}\left(\mathrm{a}_{\mathrm{i}}\right)\right\}$

- $\operatorname{Min}\left\{\lambda^{+}{ }_{\mathrm{iU}}\left(\mathrm{a}_{\mathrm{i}}\right)\right\} \geq \max \left\{\lambda^{-}{ }_{\mathrm{iU}}\left(\mathrm{a}_{\mathrm{i}}\right)\right\}$

Proof SinG G is super strong if and only if $\mathrm{D}^{-}{ }_{\mathrm{G}}<0$ and $\mathrm{D}_{\mathrm{G}}^{+}>0$ :

$$
\begin{gathered}
\sum_{\left(\mathrm{a}_{1}, \mathrm{a}_{2}\right)\left(\mathrm{b}_{1}, \mathrm{~b}_{2}\right) \in \mathrm{E}}\left(\mu_{1 \mathrm{~L}}^{+} \times \mu_{2 \mathrm{~L}}^{+}\right)\left(\left(\mathrm{a}_{1}, \mathrm{a}_{2}\right)\left(\mathrm{b}_{1}, \mathrm{~b}_{2}\right)\right)- \\
\sum_{\left(\mathrm{a}_{1}, \mathrm{a}_{2}\right)\left(\mathrm{b}_{1}, \mathrm{~b}_{2}\right) \in \mathrm{E}}\left(\mu_{1 \mathrm{~L}}^{-} \times \mu_{2 \mathrm{~L}}^{-}\right)\left(\left(\mathrm{a}_{1}, \mathrm{a}_{2}\right)\left(\mathrm{b}_{1}, \mathrm{~b}_{2}\right)\right)<0 \\
\sum_{\left(\mathrm{a}_{1}, \mathrm{a}_{2}\right)\left(\mathrm{b}_{1}, \mathrm{~b}_{2}\right) \in \mathrm{E}}\left(\mu_{1 \mathrm{~L}}^{+} \times \mu_{2 \mathrm{~L}}^{+}\right)\left(\left(\mathrm{a}_{1}, \mathrm{a}_{2}\right)\left(\mathrm{b}_{1}, \mathrm{~b}_{2}\right)\right)< \\
\sum_{\left(\mathrm{a}_{1}, \mathrm{a}_{2}\right)\left(\mathrm{b}_{1}, \mathrm{~b}_{2}\right) \in \mathrm{E}}\left(\mu_{1 \mathrm{~L}}^{-} \times \mu_{2 \mathrm{~L}}^{-}\right)\left(\left(\mathrm{a}_{1}, \mathrm{a}_{2}\right)\left(\mathrm{b}_{1}, \mathrm{~b}_{2}\right)\right)^{*}
\end{gathered}
$$

(a)
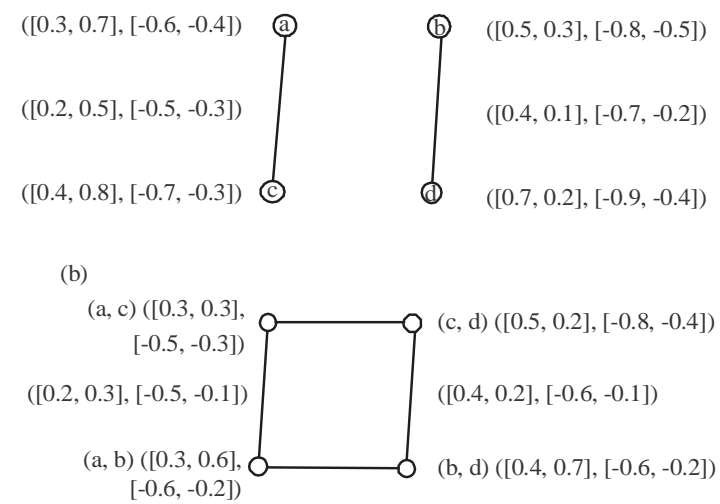

Fig. 2: Cartesian product of $G_{1}, G_{2}$

Now:

$$
\begin{gathered}
\sum_{\left(\mathrm{a}_{1}, \mathrm{a}_{2}\right)\left(\mathrm{b}_{1}, \mathrm{~b}_{2}\right) \in \mathrm{E}}\left(\mu_{\mathrm{LL}}^{+} \times \mu_{2 \mathrm{~L}}^{+}\right)\left(\left(\mathrm{a}_{1}, \mathrm{a}_{2}\right)\left(\mathrm{b}_{1}, \mathrm{~b}_{2}\right)\right)- \\
\sum_{\left(\mathrm{a}_{1}, \mathrm{a}_{2}\right)\left(\mathrm{b}_{1}, \mathrm{~b}_{2}\right) \in \mathrm{E}}\left(\mu_{\mathrm{LL}}^{-} \times \mu_{2 \mathrm{~L}}^{-}\right)\left(\left(\mathrm{a}_{1}, \mathrm{a}_{2}\right)\left(\mathrm{b}_{1}, \mathrm{~b}_{2}\right)\right)<0 \\
\sum_{\left(\mathrm{a}_{1}, \mathrm{a}_{2}\right)\left(\mathrm{b}_{1}, \mathrm{~b}_{2}\right) \in \mathrm{E}}\left(\mu_{1 \mathrm{~L}}^{+} \times \mu_{2 \mathrm{~L}}^{+}\right)\left(\left(\mathrm{a}_{1}, \mathrm{a}_{2}\right)\left(\mathrm{b}_{1}, \mathrm{~b}_{2}\right)\right)< \\
\sum_{\left(\mathrm{a}_{1}, \mathrm{a}_{2}\right)\left(\mathrm{b}_{1}, \mathrm{~b}_{2}\right) \in \mathrm{E}}\left(\mu_{1 \mathrm{~L}}^{-} \times \mu_{2 \mathrm{~L}}^{-}\right)\left(\left(\mathrm{a}_{1}, \mathrm{a}_{2}\right)\left(\mathrm{b}_{1}, \mathrm{~b}_{2}\right)\right) *
\end{gathered}
$$

Such that $\mathrm{n}=$ number of edges and $\mathrm{i}=1,2,3, \ldots, \mathrm{n}$ by the same case we get:

$$
\sum_{\left(\mathrm{a}_{1}, \mathrm{a}_{2}\right)\left(\mathrm{b}_{1}, \mathrm{~b}_{2}\right) \in \mathrm{E}}\left(\mu_{1 \mathrm{~L}}^{-} \times \mu_{2 \mathrm{~L}}^{-}\right)\left(\left(\mathrm{a}_{1}, \mathrm{a}_{2}\right)\left(\mathrm{b}_{1}, \mathrm{~b}_{2}\right)\right)=\mathrm{n}\left(\max \left\{\lambda_{\mathrm{iL}}^{-}\left(\mathrm{a}_{\mathrm{i}}\right)\right\}\right)
$$

By $*$, we get $\min \left\{\lambda^{-}{ }_{\text {iL }}\left(a_{i}\right)\right\} \geq \max \left\{\lambda^{-}{ }_{\text {iL }}\left(a_{\mathrm{i}}\right)\right\}$. Similarly, we can show $\left\{\lambda^{-}{ }_{\mathrm{iU}}\left(\mathrm{a}_{\mathrm{i}}\right)\right\} \geq \max \left\{\lambda^{-}{ }_{\mathrm{iU}}\left(\mathrm{a}_{\mathrm{i}}\right)\right\}$.

Corollary 3.9: If $G_{1}, G_{2}$ are two super strong Cartesian product graph then the Cartesian product is always super strong.

Proof: Since, $G_{1}, G_{2}$ are two super strong Cartesian product graph then $\mathrm{D}_{\mathrm{G}_{1}}^{-}<0$ and $\mathrm{D}_{\mathrm{G}_{1}}^{+}>0$ and $\mathrm{D}_{\mathrm{G}_{2}}^{-}<0$ and $\mathrm{D}_{\mathrm{G}_{2}}^{+}>0$ we know $\mathrm{D}_{\mathrm{G}_{1} \times \mathrm{G}_{2}}^{1}=\mathrm{D}_{\mathrm{G}_{1}}^{-}+\mathrm{D}_{\mathrm{G}_{2}}^{-}$and $\mathrm{D}_{\mathrm{G}_{1} \times \mathrm{G}_{2}}^{+}=\mathrm{D}_{\mathrm{G}_{1}}^{+}+\mathrm{D}_{\mathrm{G}_{2}}^{+}$for the Cartesian product of bipolar interval valued fuzzy graph. By theorem 3.7 we get .

$\mathrm{D}_{\mathrm{G}_{1} \times \mathrm{G}_{2}}^{-}<0$ and $\mathrm{D}_{\mathrm{G}_{1} \times \mathrm{G}_{2}}^{+}>0$.

Lemma 3.10: If $G_{1}, G_{2}$ are two very week Cartesian product graph then $G_{1} \times G_{2}$ is always very week.

Proof: Straight forward 
Definition 3.11: For any vertex $\left(a_{1}, a_{2}\right) \in V_{1} \otimes V_{2}$ then the degree of a vertex in tensor product is:

$$
\begin{aligned}
& D_{\mathrm{G}_{1} \otimes \mathrm{G}_{2}}^{-}\left(\begin{array}{c}
\mathrm{a}_{1}, \\
\mathrm{a}_{2}
\end{array}\right)=\sum_{\left(\mathrm{a}_{1}, \mathrm{a}_{2}\right)\left(\mathrm{b}_{1}, \mathrm{~b}_{2}\right) \in \mathrm{E}}\left(\mu_{1 \mathrm{~L}}^{+} \otimes \mu_{2 \mathrm{~L}}^{+}\right)\left(\left(\mathrm{a}_{1}, \mathrm{a}_{2}\right)\left(\mathrm{b}_{1}, \mathrm{~b}_{2}\right)\right)- \\
& \sum_{\left(\mathrm{a}_{1}, \mathrm{a}_{2}\right)\left(\mathrm{b}_{1}, \mathrm{~b}_{2}\right) \in \mathrm{E}}\left(\mu_{1 \mathrm{~L}}^{-} \otimes \mu_{2 \mathrm{~L}}^{-}\right)\left(\left(\mathrm{a}_{1}, \mathrm{a}_{2}\right)\left(\mathrm{b}_{1}, \mathrm{~b}_{2}\right)\right)= \\
& \sum_{\mathrm{a}_{1}, \mathrm{~b}_{\mathrm{L}} \in \mathrm{E}_{1}, \mathrm{a}_{2}, \mathrm{~b}_{2} \in \mathrm{E}_{2}} \min \left\{\mu_{1 \mathrm{~L}}^{+}\left(\mathrm{a}_{1} \mathrm{~b}_{1}\right), \mu_{2 \mathrm{~L}}^{+}\left(\mathrm{a}_{2} \mathrm{~b}_{2}\right)\right\}- \\
& \sum_{\mathrm{a}_{1}, \mathrm{~b}_{\mathrm{L}} \in \mathrm{E}_{\mathrm{1}}, \mathrm{a}_{2}, \mathrm{~b}_{2} \in \mathrm{E}_{2}} \max \left\{\mu_{1 \mathrm{~L}}^{-}\left(\mathrm{a}_{1} \mathrm{~b}_{1}\right), \mu_{2 \mathrm{~L}}^{-}\left(\mathrm{a}_{2} \mathrm{~b}_{2}\right)\right\}
\end{aligned}
$$

Definition 3.12: For any vertex $\left(a_{1}, a_{2}\right) \in V_{1} * V_{2}$ then the degree of a vertex in lexicographic product is:

$$
\begin{aligned}
& \mathrm{D}_{\mathrm{G}_{1}{ }^{*} \mathrm{G}_{2}}^{-}=\sum_{\left(\mathrm{a}_{1}, \mathrm{a}_{2}\right)\left(\mathrm{b}_{1}, \mathrm{~b}_{2}\right) \in \mathrm{E}}\left(\mu_{1 \mathrm{~L}}^{+} \times \mu_{2 \mathrm{~L}}^{+}\right)\left(\left(\mathrm{a}_{1}, \mathrm{a}_{2}\right)\left(\mathrm{b}_{1}, \mathrm{~b}_{2}\right)\right)- \\
& \sum_{\left(\mathrm{a}_{1}, \mathrm{a}_{2}\right)\left(\mathrm{b}_{1}, \mathrm{~b}_{2}\right) \in \mathrm{E}}\left(\mu_{1 \mathrm{~L}}^{-} \times \mu_{2 \mathrm{~L}}^{-}\right)\left(\left(\mathrm{a}_{1}, \mathrm{a}_{2}\right)\left(\mathrm{b}_{1}, \mathrm{~b}_{2}\right)\right)= \\
& \max \left\{\sum_{\substack{a_{1}=b_{1}, a_{2} b_{2} \in E_{2}}} \min \left\{\begin{array}{l}
\lambda_{1 \mathrm{~L}}^{+}\left(\mathrm{a}_{1}\right), \\
\mu_{2 \mathrm{~L}}^{+}\left(a_{2} b_{2}\right)
\end{array}\right\}, \sum_{\substack{\mathrm{a}_{2}=\mathrm{b}_{2}, a_{1} \mathrm{~b}_{1} \in \mathrm{E}_{1}}} \min \left\{\begin{array}{l}
\lambda_{1 \mathrm{~L}}^{+}\left(\mathrm{a}_{2}\right), \\
\mu_{1 \mathrm{~L}}^{+}\left(\mathrm{a}_{1} \mathrm{~b}_{1}\right)
\end{array}\right\}\right\} \text { - } \\
& \max \left\{\sum_{\substack{a_{1}=b_{1}, a_{2} b_{2} \in E_{2}}} \max \left\{\begin{array}{l}
\lambda_{1 \mathrm{~L}}^{-}\left(a_{1}\right), \\
\mu_{2 \mathrm{~L}}^{-}\left(a_{2} b_{2}\right)
\end{array}\right\}, \sum_{\substack{a_{2}=b_{2}, a_{1} b_{1} \in E_{1}}} \max \left\{\begin{array}{l}
\lambda_{i L}^{-}\left(a_{2}\right), \\
\mu_{1 L}^{-}\left(a_{1} b_{1}\right)
\end{array}\right\}\right\}
\end{aligned}
$$

\section{CONCLUSION}

In this study, we introduce the degree and discuss it for Cartesian product, tensor product and lexicographic product of two bipolar interval valued of fuzzy graphs also we can generalized it to like strong product, week product. We use this concept in homomorphism bipolar interval valued fuzzy graph and in bipolar intuitionistic interval valued fuzzy graph on the other hand the concept of fuzzy sets, bipolar fuzzy sets and intuitionistic fuzzy sets will be applied to the following topics listed by Alnaser (2014a, b, 2017, 2018).

\section{REFERENCES}

Akram, M. and W.A. Dudek, 2011. Interval-valued fuzzy graphs. Comput. Math. Appl., 61: 289-299.

Alnaser, A.M., 2014b. Set-theoretic foundations of the modern relational databases: Representations of table algebras operations. Br. J. Math. Comput. Sci., 4: 3286-3293.

Alnaser, A.M., 2014a. Streaming algorithm for multi-path secure routing in mobile networks. IJCSI. Intl. J. Comput. Sci. Issues, 11: 112-114.

Alnaser, A.M., 2017. A method of forming the optimal set of disjoint path in computer networks. J. Appl. Comput. Sci. Math., 11: 9-12.
Alnaser, A.M., 2018. A method of multipath routing in SDN networks. Adv. Comput. Sci. Eng., 17: 11-17.

Massa'deh, M.O. and A. Ba'arah, 2013. Some contribution on isomorphic fuzzy graphs. Adv. Applic. Discrete Math., 11: 199-206.

Massa'deh, M.O. and N.K. Gharaibeh, 2011. Some properties on fuzzy graphs. Adv. Fuzzy Math., 6: 245-252.

Massa'deh, M.O., 2010a. A note on upper fuzzy subrings and upper fuzzy ideals. S. East Asian J. Math. Math. Sci., 9: 41-49.

Massa'deh, M.O., 2010b. Short communicationsome properties of upper fuzzy order. Afr. J. Math. Comput. Sci. Res., 3: 192-194.

Massa'deh, M.O., 2017. On bipolar fuzzy cosets, bipolar fuzzy ideals and isomorphisms of $\gamma$-near rings. Far East J. Math. Sci., 102: 731-747.

Mishra, S.N. and A. Pal, 2016. Bipolar interval valued fuzzy graphs. Intl. Sci., 9: 1022-1028.

Muthuraj, R. and M. Sridharan, 2012. Bipolar anti fuzzy HX group and its lower level sub HX groups. J. Phys. Sci., 16: 157-169.

Muthuraj, R., M. Sridharan and K.H. Manikandan, 2016. Some properties of bipolar fuzzy normal HX subgroup and its normal level sub HX groups. Global Res. Dev. J. Eng., 2: 57-65.

Ramprasad, N. Srinivasarao and S. Satyanarayana, 2016. A study on interval-valued fuzzy graphs. Comput. Sci. Telecommun., 50: 60-72.

Ramya, S. and S. Lavanya, 2017. Edge contraction on bipolar fuzzy graphs. Intl. J. Trend Res. Dev., 4: 475-477.

Rashmanlou, H. and M. Pal, 2013b. Antipodal interval valued fuzzy graphs. Intl. J. Appl. Fuzzy Sets Artif. Intell., 3: 107-130.

Rashmanlou, H. and M. Pal, 2013a. Balanced interval-valued fuzzy graphs. J. Phys. Sci., 17: 43-57.

Rashmanlou, H. and M. Pal, 2013. Some properties of highly irregular interval-valued fuzzy graphs. World Appl. Sci. J., 27: 1756-1773.

Rashmanlou, H. and Y.B. Jun, 2013. Complete interval-valued fuzzy graphs. Ann. Fuzzy Math. Inf., 6: 677-687.

Zadeh, L.A., 1965. Fuzzy sets. Inform. Control, 8: 338-353.

Zhang, W.R., 1998. (Yin) (Yang) bipolar fuzzy sets. Proceedings of the 1998 IEEE International Conference on Fuzzy Systems and IEEE World Congress on Computational Intelligence (Cat. No. 98CH36228) Vol. 1, May 4-9, 1998, IEEE, Anchorage, Alaska, USA., pp: 835-840. 